\title{
Tuberculosis lymph node granulomas: using transcriptomics to discover immunopathology paradigms and guide host-directed therapy
}

\author{
James J. Phelan, Seónadh O'Leary, and Joseph Keane \\ TB Immunology Group, Department of Clinical Medicine, Trinity Translational Medicine Institute, Trinity College Dublin, Dublin, Ireland.
}

\begin{abstract}
Immunometabolism is a burgeoning field of investigation in tuberculosis host defense, susceptibility, and pathophysiology. Unbiased approaches to studying tuberculosis have, as expected, confirmed that pathways of immunometabolism are crucial in these disease processes. In this issue of the $J C I$, Reichmann et al. studied carefully controlled human lymph node tuberculosis and uncovered Sphingosine kinase 1 as a druggable target of interest that could support the infected host. Future host-directed therapy research might seek to establish the different cellular consequences of sphingolipid pathway manipulation. Animal models will be especially useful to establish the role of this pathway, which might target diseased organs to improve mycobactericidal effect and limit pathology.
\end{abstract}

Transcriptomics: first unbiased steps to host-directed therapy Unbiased methodologies, such as wellcontrolled transcriptomics, hold great promise for dissecting tuberculosis host defense and immunopathology pathways. For example, the bioinformatic analysis of Berry et al. emphasized a central role of Type I interferons, which are now an important focus for research in tuberculosis (TB) immunity (1). At their best, these big data methods reveal new pathways or confirm the importance of known pathways, like autophagy (2). While transcriptomics hold great promise in developing diagnostics (3), there is also a promise that these large analyses can direct the development of host-directed therapy (HDT). It is important to remember that HDT in TB can also include driving pathways that improve Mycobacterium tuberculosis (Mtb) killing as well as the traditional notion that TB HDT is used with the intent to diminish inflammation (3). Although many papers have published gene polymorphism and transcriptomics profiles that are TB associated, there are few studies that perform the mechanistic experiments needed to show that a distinct pathway is important in the host response (4).

In this issue of the JCI, Reichmann et al. do just the sort of mechanistic experiments required to elucidate a host response pathway (5). The researchers exploited their access to neck and mediastinum lymph node from subjects with TB or sarcoidosis, using sarcoidosis noninfectious granulomas as a control. This comparison allowed the authors to identify a panel of druggable intracellular targets, which emerged after analysis, as candidates specifically associated with lymph node TB. After the researchers manipulated 12 enzymes, inhibiting Sphingosine kinase 1 (Sphk1) showed a mechanistic role in controlling Mtb growth, diminishing intracellular $\mathrm{pH}$,

Related Article: https://doi.org/10.1172/JCl148136

Conflict of interest: The authors have declared that no conflict of interest exists.

and suppressing inflammatory mediators in cellular models of infection.

\section{The power of $3 \mathrm{D}$ models for TB}

One of the most common models used to study TB host defense is the infection of isolated macrophages with the Mtb bacillus at different multiplicities of infection. This useful system allows for the controlled dissection of the initial host-pathogen interaction. Notably, the assay may serve to recreate host events that occur generally in infection and disease, and also in the human lung (maybe $80 \%$ of the time after natural Mtb infection), namely the process of early clearance (6). However, patients with disease have a complex immune phenotype that is also influenced by local environmental factors, such as $\mathrm{pH}$, lactate level, and the lack of glucose. Consequently, the research field has responded by developing 3D cellular systems, which might better model disease, to study pathways of immunopathology (7). These approaches include in vitro human granuloma models, bioelectrospray 3D model (used by Reichmann et al.; ref. 5), and what is known as the "organ on a chip" model (8). It is an important finding in Reichmann et al. (5), though not surprising, that the 3D collagen model of infected PBMCs more closely resembles the gene expression profile of lymph node disease when compared with the 2D model. The authors used the 3D model to identify druggable targets of interest that were $\mathrm{TB}$ specific. They then returned to the 2D model to show the importance of sphingolipid metabolism in the host response and tissue-destruction signals.

COVID-19 research has brought into focus the importance of rapid HDT development in treating pathogens for which there are poor antimicrobial options (9). By introducing another paradigm of host defense, centered on ceramide metabolism, the 


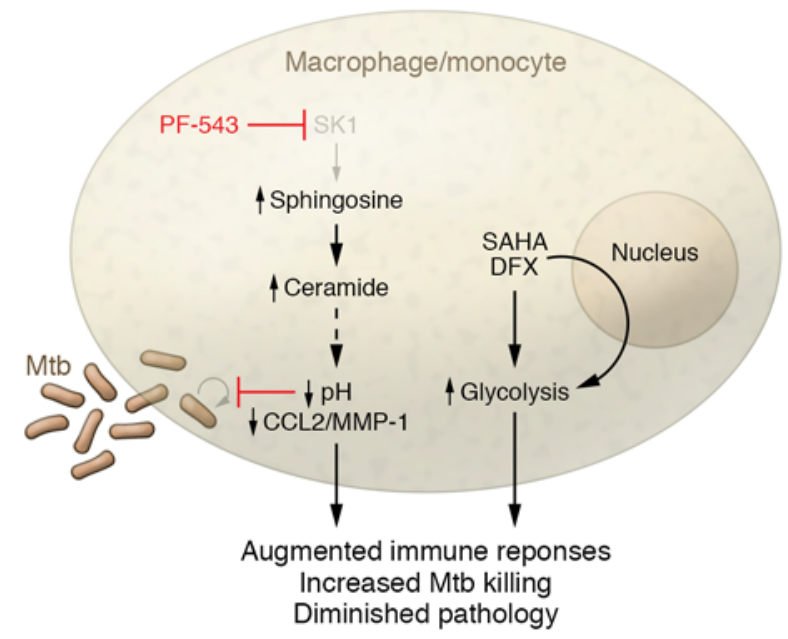

Figure 1. Sphingolipid metabolism as a viable HDT option. Identifying additional HDT options, such as DFX and SAHA, through the manipulation of sphingolipid metabolism could be a promising approach in the treatment of TB. Reichmann et al. (5) targeted macrophage/monocyte sphingosine metabolism by inhibiting Sphk1 with PF-543. Sphk1 inhibition controlled Mtb growth, reduced intracellular pH in infected monocytes, and suppressed secretion of CCL2 and MMP-1.

Reichmann et al. study can direct research that might develop HDT for TB (5). It may seem obvious that important cellular processes (such as cell death, autophagy, and metabolism) play a crucial role in TB, but the challenge is to manipulate these processes at sites of disease without systemic toxic effects. Inhaled therapies might avoid systemic side effects and are an option for pulmonary disease (10), but they are unlikely to help in lymphadenitis.

\section{Immunometabolic HDT is a fast-evolving discipline}

To date, the development of immunometabolic HDT has primarily focused on targeting pathways central to glucose metabolism, more specifically glycolysis and oxidative phosphorylation, to combat Mtb infection in the human lung. We have seen that existing agents can safely modify immunometabolic pathways to favor myeloid pathways that support the host in TB to potentially benefit infected host cells. For example, some HDTs have been shown to target these immunometabolic pathways during early Mtb infection in primary human macrophages to enhance early Mtb clearance by augmenting glycolysis (Figure 1). One such approach to Mtb infection involved targeting epigenetic components with suberanilohydroxamic acid (SAHA), specifically histone deacetylases, in human macrophages to enhance glycolysis, immune function, and downstream $\mathrm{T}$ helper cell responses (11). Another approach has been to support the modulation of glucose metabolism by regulating iron metabolism. The iron chelator desferrioxamine (DFX) enhances glycolytic metabolism and augments host immune function in human macrophages during early Mtb infection by switching on the master immunometabolic regulator HIF1 $\alpha$ (12), which helps to enhance killing of Mtb in combination with the TB antimicrobial bedaquiline (13). Some evidence suggests that existing anti-TB drugs could encompass some potential at modulating these key immunometabolic pathways (14). Omics approaches have emphasized itaconate as a potential targetable intermediate metabolite in inflammation and infectious diseases (15).

\section{Sphingolipid metabolism as a reasonable target for HDT}

Emerging insights into sphingolipid metabolism pathways may also identify an array of HDT options, in conjunction with the renewed interest in immunometabolism in general, for the treatment of a variety of respiratory infections, including Mtb (16). Sphingolipids are a major constituent of the mucus secreted by epithelial cells in the lung and can be readily converted from one bioactive metabolite to another, encompassing both structural and functional roles. More pertinently, the phagocytotic process undertaken by alveolar macrophages during early Mtb infection fundamentally depends on sphingomyelin biosynthesis to enable host cell entry (17). The sphingolipid sphingosine-1 phosphate (S-1P) is involved in the induction of antimicrobial activity in human macrophages, resulting in the intracellular killing of nonpathogenic Mycobacterium smegmatis and pathogenic Mtb (18). Recently, however, S-1P has been shown to mediate the expression of iNOS proteins in macrophages, their polarization toward the M1 phenotype, and their secretion of IFN- $\gamma$, while also enhancing the infiltration of pulmonary $\mathrm{CD}_{11} \mathrm{~b}^{+}$macrophages in murine lungs during the course of Mtb infection (19). Now, Reichmann et al. (5) build on this knowledge and mechanistically link the sphingolipid metabolite Sphk1 with the expression of CCL2 and MMP-1 using 2D flat and 3D cell culture models of Mtb infection (Figure 1). However, the definition of how sphingolipid metabolic pathways effect TB immunity still remains incomplete. The possibility of manipulating sphingolipid/ceramide metabolism, for example, to favor ceramide accumulation, is a hypothesis worth pursuing in human and animal knock-out models. Genedeficient models might identify the rate-limiting processes of sphingolipid metabolism, which underpins host susceptibility to Mtb infection.

\section{Acknowledgments}

This work was funded by the Royal City of Dublin Hospital Trust.

Address correspondence to: Joseph Keane, St. James's Hospital, Dublin 8, Dublin, Ireland. Phone: 353.1.410.3920; Email: josephmk@tcd.ie. 
1. Berry MP, et al. An interferon-inducible neutrophil-driven blood transcriptional signature in human tuberculosis. Nature. 2010;466(7309):973-977.

2. Kumar D, et al. Genome-wide analysis of the host intracellular network that regulates survival of Mycobacterium tuberculosis. Cell. 2010;140(5):731-743.

3. Singhania A, et al. The value of transcriptomics in advancing knowledge of the immune response and diagnosis in tuberculosis. Nat Immunol. 2018;19(11):1159-1168.

4. Cheallaigh $\mathrm{CN}$, et al. A common variant in the adaptor mal regulates interferon gamma signaling. Immunity. 2016;44(2):368-379.

5. Reichmann MT, et al. Integrated transcriptomic analysis of human tuberculosis granulomas and a biomimetic model identifies therapeutic targets. J Clin Invest. 2021;131(15):e148136.

6. Israel HL, et al. A study of tuberculosis among students of nursing. JAMA. 1941;117:461-473.

7. Tezera LB, et al. Dissection of the host-pathogen interaction in human tuberculosis using a bioengineered 3-dimensional model. Elife. 2017;6:e21283.

8. Elkington $\mathrm{P}$, et al. In vitro granuloma models of tuberculosis: potential and challenges. J Infect Dis. 2019;219(12):1858-1866.

9. Recovery Collaborative Group. Dexamethasone in hospitalized patients with Covid-19. N Engl J Med.2021;384(8):693-704.

10. O'Connor G, et al. Inhalable poly(lactic-coglycolic acid) (PLGA) microparticles encapsulating all-trans-Retinoic acid (ATRA) as a hostdirected, adjunctive treatment for Mycobacterium tuberculosis infection. Eur J Pharm Biopharm. 2019;134:153-165.

11. Cox DJ, et al. Inhibiting histone deacetylases in human macrophages promotes glycolysis, IL-1 $\beta$, and T helper cell responses to Mycobacterium tuberculosis. Front Immunol. 2020;11:1609.

12. Phelan JJ, et al. Desferrioxamine supports metabolic function in primary human macrophages infected with Mycobacterium tuberculosis. Front Immunol. 2020;11:836.

13. Cahill C, et al. The iron chelator desferrioxamine increases the efficacy of bedaquiline in primary human macrophages infected with BCG. Int J
Mol Sci. 2021;22(6):2938

14. Cahill C, et al. Understanding and exploiting the effect of tuberculosis antimicrobials on host mitochondrial function and bioenergetics. Front Cell Infect Microbiol. 2020;10:493.

15. Mills EL, et al. Itaconate is an anti-inflammatory metabolite that activates Nrf2 via alkylation of KEAP1. Nature. 2018;556(7699):113-117.

16. Sharma L, H Prakash. Sphingolipids are dual specific drug targets for the management of pulmonary infections: perspective. Front Immunol. 2017;8:378.

17. Niekamp P, et al. Sphingomyelin biosynthesis is essential for phagocytic signaling during mycobacterium tuberculosis host cell entry. mBio. 2021;12(1):e03141-20.

18. Garg SK, et al. Sphingosine 1-phosphate induces antimicrobial activity both in vitro and in vivo. J Infect Dis. 2004;189(11):2129-2138.

19. Nadella V, et al. Sphingosine-1-phosphate (S-1P) promotes differentiation of naive macrophages and enhances protective immunity against Mycobacterium tuberculosis. Front Immunol. 2019;10:3085. 